Article

\title{
Thermal Cloaking in Nanoscale Porous Silicon Structure by Molecular Dynamics
}

\author{
Jian Zhang, Haochun Zhang *(D), Yiyi Li, Qi Wang and Wenbo Sun
}

check for updates

Citation: Zhang, J.; Zhang, H.; Li, Y.; Wang, Q.; Sun, W. Thermal Cloaking in Nanoscale Porous Silicon Structure by Molecular Dynamics. Energies 2022, 15, 1827. https://doi.org/ $10.3390 /$ en15051827

Academic Editors: Moghtada Mobedi and Kamel Hooman

Received: 6 February 2022

Accepted: 25 February 2022

Published: 2 March 2022

Publisher's Note: MDPI stays neutral with regard to jurisdictional claims in published maps and institutional affiliations.

Copyright: (C) 2022 by the authors. Licensee MDPI, Basel, Switzerland. This article is an open access article distributed under the terms and conditions of the Creative Commons Attribution (CC BY) license (https:// creativecommons.org/licenses/by/ $4.0 /)$.
School of Energy and Engineering, Harbin Institute of Technology, Harbin 150001, China; 20b902063@stu.hit.edu.cn (J.Z.); 18b902056@stu.hit.edu.cn (Y.L.); 21s002039@stu.hit.edu.cn (Q.W.); 21s102109@stu.hit.edu.cn (W.S.)

* Correspondence: hczhang@hit.edu.cn

\begin{abstract}
Nanoscale thermal cloaks have great potential in the thermal protection of microelectronic devices, for example, thermal shielding of thermal components close to the heat source. Researchers have used graphene, crystalline silicon film, and silicon carbide to design a variety of thermal cloaks in different ways. In our previous research, we found that the porous structure has lower thermal conductivity compared to bulk silicon; thus, so we tried to use the porous structure to construct the functional region to control the heat flux. We first calculated the thermal conductivity of crystalline silicon and porous silicon films by means of nonequilibrium molecular dynamics, proving that the porous structure satisfied the conditions for building a thermal cloak. A rectangular cloak with a porous structure was constructed, and a crystalline silicon film was used as a reference to evaluate its performance by the index of the ratio of thermal cloaking. We found that the thermal cloak built with a porous structure could produce an excellent cloaking effect. Lastly, we explain the mechanism of the cloaking phenomenon produced by a porous structure with the help of phonon localization theory. Porous structures have increased porosity compared to bulk silicon and are not conducive to phonon transport, thus producing strong phonon localization and reducing thermal conductivity. Our research expands the construction methods of nanocloaks, expands the application of porous structure materials, and provides a reference for the design of other nanodevices.
\end{abstract}

Keywords: porous silicon; thermal cloak; phonon localization; molecular dynamics; nanoscale

\section{Introduction}

The manipulation and regulation of heat flux has always been a research hotspot, and the transformation thermotics theory [1] and thermal metamaterials have further promoted its development. Researchers have realized the regulation of heat flux. For example, the thermal cloak [2-11] is a thermal function device that protects the objects in the functional region from the outside temperature without disturbing the background temperature field. Hu et al. [12] put forward the concept of "inverse heat cloak", whereby the heat flux is successfully concentrated to the functional region to achieve local heating, also known as the thermal concentrator [13]. In addition, researchers have designed a thermal rotator [14], thermal camouflage [15-21], thermal illusion [22-25], encrypted thermal printing [26], etc. and conducted experimental verification. So far, the regulation of heat flux has been achieved in various situations on the macroscale. This has also greatly promoted the development of thermodynamics and put forward different thermodynamic evaluation indicators, such as local entropy production rate [27], and response entropy [28], thus also better evaluating the effect of heat flux regulation and providing a powerful tool for performance optimization of heat flux regulation devices. To advance the application of heat flux control devices in industrial production, Li et al. [29] evaluated the cloaking performance and environmental response of a 2D thermal cloak based on a dynamic environment in the form of sinusoids, using variables such as ambient amplitude and temperature difference. 
Considering the heat dissipation and energy loss of the whole process, the local entropy generation rate was introduced to analyze the influence of environmental parameters on the cloaking system. The results showed that, in the functional region, the thermal dissipation and irreversible energy loss of the system were caused by the difference in thermal conductivity between adjacent layers, and the heat dissipation capacity of the thermal cloak increased with the increase in the ambient amplitude and temperature difference. According to the distribution of entropy yield, it was found that thermal cloaking has a corresponding application range for different environmental variables. Considering the requirements for different energy types and functions in practical applications, research on metamaterial structures that simultaneously realize complex tuning functions in multiphysics is particularly important. Xu et al. [30] solved the complex regulation problem in multiphysics by designing a discrete array of "sources" using the "rotation-linear" mapping method. Discrete arrays of "sources" were designed to reconfigure each physical field produced by a single excitation source and form a "multiple source" array field distribution. This research provided a new way to efficiently manipulate and distribute the energy generated by excitation sources, which could lead to the development of solar cells and thermoelectric devices. Li et al. [31] designed and built an intelligent flux transfer regulator with uniformly distributed conduction parameters and efficient operation in both thermal and DC electric fields. In addition, with the help of the discretization method, effective medium theory, and other control devices, the effectiveness of the established device was verified experimentally. This work not only further develops the diversity and applicability of energy regulation methods, but also provides a reference for studying energy transport phenomena from different disciplines. However, there are still great challenges to achieve complete thermal cloaking in practical experiments.

In recent years, due to the miniaturization of electronic equipment, the heat flux density of electronic devices has become larger and larger, and nanoscale heat flux control has become particularly important. Phonons, as micro/nanoscale heat transfer carriers, have unique characteristics of wave-particle duality. Researchers have designed thermal diodes [32,33], the phonon Hall effect [34-36], thermal rectification [37-40], etc. with the help of molecular dynamics and other means. The commonly used means for thermal protection of electronic devices is to use physical isolation for thermal shielding of thermal components close to the heat source. The research on the nanoscale thermal cloak is very important. Ye et al. [41] first used a graphene film to construct a nanocloak of chemical functionalization. The partial chemical functionalization on graphene was used to form heat flux channels that avoid specific regions. Due to the phonon localization, the heat flux automatically avoided the transition region, and the central part was protected from the heat flux. Considering the popularity of graphene, this study not only has important application value for thermal protection of graphene-based devices, but also has far-reaching implications for the development of other nanoelectronic devices. Liu et al. [42] used a crystalline silicon film with the "melting-quenching" technique to build a nanocloak. The results showed that the thermal cloaking effect was proportional to the width of the cloak ring. By exploring the underlying mechanism of nanoscale thermal cloaking by computing PDOS and MPR, it was found that phonon localization occurred in the nanocloak ring region and was responsible for the reduced thermal conductivity of amorphous silicon. The proposed method to engineer nanocloaks by in situ tuning of the material's atomic lattice structure is more practical and efficient, and it may open the way for nanoscale thermal functions and thermal management in nanophotonics and nanoelectronics. Choe et al. [43] designed a particle irradiation platform and observed the phenomenon of thermal cloaking experimentally. The ability to control microscale heat flux using this platform provides an opportunity to explore new ideas for microscopic thermal management. Furthermore, the demonstrated ion-writing microcaloritics has the potential to be a versatile platform for controlling heat flow at the microscale, similar to what nanofluids do to fluids. In addition, we built a nanocloak by perforating a crystalline silicon film [44-46], while the effects of the number, size, and arrangement of perforations on its performance were explored. The 
results showed that the nanocloak designed by the perforation method could also show a good cloaking effect, and the cloaking effect was positively correlated with the number and size of the holes. When the number and size of holes were fixed, the best arrangement was circular. The nanocloak performance was optimized using the response surface method, and the fitting equations of multiple influencing factors were obtained. The best parameter selection interval was obtained by analyzing the interaction between two different parameters. By selecting the parameters in the best interval, the best cloaking effect could be obtained. The dynamic response and the effect of concave depth on its cloaking performance were studied, and the functional relationships of response temperature and the ratio of thermal cloaking with concave depth were obtained. The results showed that, under the condition of ensuring the stability of the structure, a greater depth led to better cloaking performance. Xiao et al. [47] used nanoporous thin films to realize the thermal cloaking. This work presented periodic square nanoholes and rectangular nanogrooves as efficient methods to locally tune the thermal conductivity and thermal anisotropy of thin films and potentially atomically thick materials. Thermal cloaking efficiencies were calculated and compared with theoretical predictions of continuous thermal conductivity distributions. This new approach enables two-dimensional nanocloaking of thin-film devices by eliminating the challenges of fabricating nanocomposites with well-controlled thermal properties. The periodic arrangement of the porous structure can be equivalent to a phononic crystal. According to the research of Chen et al. [48], the porosity has a great influence on the thermal conductivity of the phononic crystal. A greater porosity leads to a lower thermal conductivity, because a larger porosity corresponds to larger nanocubic pores, thereby narrowing the path for phonon transmission and increasing the surface-to-body ratio of the system, which leads to stronger structural reflections and, ultimately, lower thermal conductivity. No thermal cloak based on a porous structure has been found. Therefore, we used a porous structure to build a thermal cloak in this research.

In this paper, on the basis of nonequilibrium dynamics (NEMD), we calculate the thermal conductivity of porous structures and crystalline silicon films, and we use the porous structure to build a rectangular nanocloak. According to the index of the ratio of thermal cloaking, we find that the porous structure can be used to build a nanocloak to produce an excellent cloaking effect. Furthermore, the heat flux and temperature distribution are drawn to intuitively show the cloaking phenomenon. In addition, we explain the mechanism of the cloaking phenomenon produced using a porous structure with the help of phonon localization theory. Porous structures have increased porosity compared to bulk silicon and are not conducive to phonon transport, thus producing strong phonon localization and reducing the thermal conductivity. Our research can promote the application of porous materials and expand the construction methods of nanocloaks. Section 2 introduces the model and the basic theory of numerical simulation, Section 3 analyzes the simulation results, and Section 4 introduces the main conclusions of this paper. Figure 1 shows the overall calculation flow of this paper.

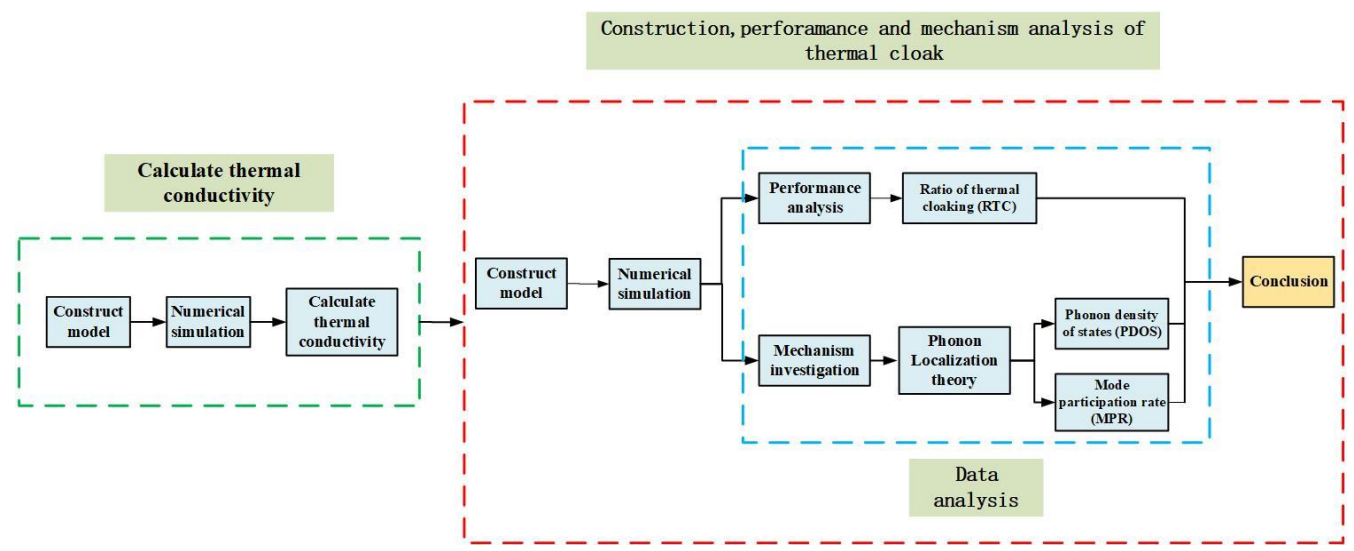

Figure 1. The detailed calculation process of this paper. 


\section{Model and Methodology}

\subsection{Construction of Computational Models}

In this paper, the material we chose was crystalline silicon with a lattice constant of $5.431 \AA$ A. The basic unit constituted a unit cell (UC). We used LAMMPS [49] to construct the model and conduct the simulation. The built-in modeling commands of LAMMPS were used for modeling. First, a silicon film plate model was constructed as a calculation reference, and then a porous structure was built on the plate. Since the number of regions is limited in LAMMPS, we created it in two steps. The first step was to delete the plate $x$ - and $y$-direction atoms, as shown in Figure $2 \mathrm{~b}$, while the second step was to delete the $z$-direction atoms. The thermal conductivity calculation model is shown in Figure 2 . The size of the simulation box was $50 \times 12 \times 4 \mathrm{UC}$.

(a)

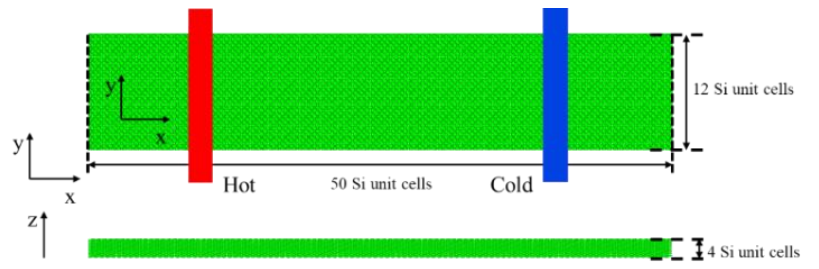

(b)

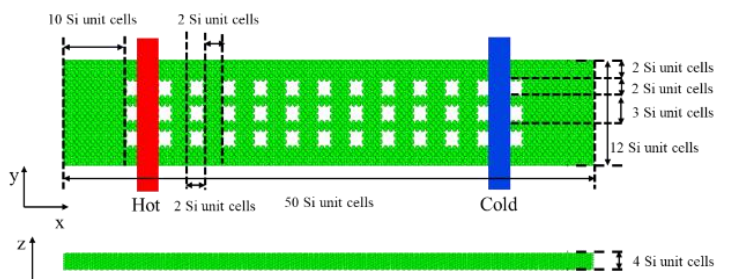

Figure 2. The thermal conductivity calculation model: (a) crystalline film; (b) porous structure film.

Since the porous structure selected in this paper was a cubic nanoporous structure, it was not easy to build a classic circular nanocloak. Therefore, according to our previous research experience, we selected a more reasonable scale to build a porous rectangular thermal cloak. The calculation model of the nanocloak is shown in Figure 3. The model size was $60 \times 40 \times 4 \mathrm{UC}$; the functional region was constructed with a porous structure, while the remainder was crystalline silicon. The two ends were fixed regions to prevent the whole model from moving, and the length was 2 UC. The thermostat region was divided into cold and hot baths, both of which were $8 \mathrm{UC}$ in length. The remaining regions were background regions.

\subsection{Methodology}

In this research, the Verlet algorithm [50] was used to solve Newton's equations of motion, as it is the most accurate velocity calculation method. The canonical ensemble (NVT) and the microcanonical ensemble (NVE) were used in the simulation. The correctness of the molecular dynamics simulation results depends on the accuracy of the potential function. According to our previous research, we chose the Tersoff potential [51].

$$
E=\sum_{i} E_{i}=\frac{1}{2} \sum_{i \neq j} f_{C}\left(r_{i j}\right)\left[f_{R}\left(r_{i j}\right)+b_{i j} f_{A}\left(r_{i j}\right)\right],
$$

where $E$ is the total energy, $i$ and $j$ are atom labels, $r$ is the distance between atoms, $f$ is the potential function, $C$ is the cutoff function, $R$ and $A$ are repulsive and attractive pairs, respectively, and $b$ is the bond order between atoms.

\subsubsection{Thermal Conductivity Calculation}

The key to design a nanocloak is to construct a functional region with low thermal conductivity; thus, calculating the thermal conductivity is crucial for successful construction. There are two commonly used methods, EMD and NEMD. We chose the NEMD method, which is also known as the "nonequilibrium" method. The so-called nonequilibrium state is a state opposite to the equilibrium state. In most molecular dynamics simulations, relaxation is required. Relaxation is also called the "running equilibrium", whereby the shape of the material in the equilibrium state is obtained, while the temperature and energy of the system remain basically unchanged. The nonequilibrium state is the opposite, 
whereby the temperature is not constant, but maintains a certain gradient. We can calculate the lattice thermal conductivity as follows:

$$
J=-\kappa \nabla T,
$$

where $J$ is the given heat flux, $\nabla T$ is the temperature gradient, and $\kappa$ is a tensor [52-55]. Our calculated thermal conductivity was $\kappa_{x x}$. Since the simulation was mainly in the $x y$ plane, $\kappa_{z z}$ was ignored, periodic boundary conditions were used in the simulation process, and the model was symmetrically distributed; hence, in the $x y$ plane, thermal conductivity was isotropic, $\kappa_{x x}=\kappa_{y y}$.
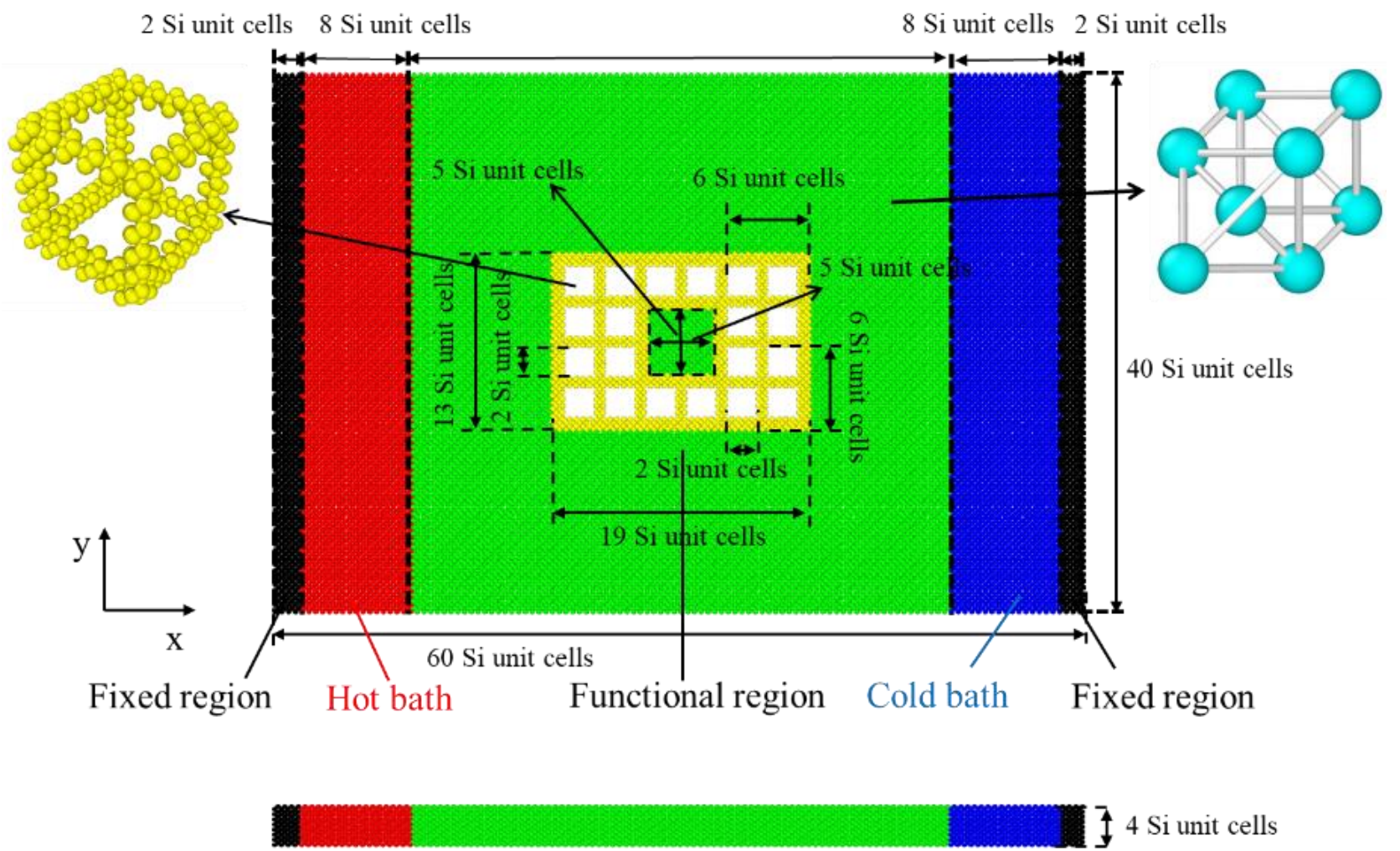

Figure 3. Schematic diagram of each region of the nanocloak.

\subsubsection{Heat Flux and Temperature Calculation}

In order to evaluate the cloaking performance of the nanocloak and visually display the cloaking phenomenon, referring to [56], we can calculate the single-atom heat flux as follows:

$$
\mathbf{J}=\frac{1}{V}\left[\sum_{i} e_{i} \mathbf{v}_{i}-\sum_{i} \mathbf{S}_{i} \mathbf{v}_{i}\right]
$$

where $\mathbf{J}$ is the heat flux, $V$ is the volume, $i$ is an atomic label, $e$ is the total energy, which includes kinetic and potential energy, $\mathbf{v}$ is the velocity vector, and $\mathbf{S}$ is the pressure tensor. In all three directions, the heat flux can be described as follows:

$$
\begin{aligned}
& J_{x}=\frac{1}{V}\left[\sum_{i} e_{i} v_{x i}-\sum_{i}\left(S_{i x x} v_{i x}+S_{i x y} v_{i y}+S_{i x z} v_{i z}\right)\right] \\
& J_{y}=\frac{1}{V}\left[\sum_{i} e_{i} v_{y i}-\sum_{i}\left(S_{i y x} v_{i x}+S_{i y y} v_{i y}+S_{i y z} v_{i z}\right)\right]
\end{aligned}
$$




$$
J_{z}=\frac{1}{V}\left[\sum_{i} e_{i} v_{z i}-\sum_{i}\left(S_{i z x} v_{i x}+S_{i z y} v_{i y}+S_{i z z} v_{i z}\right)\right]
$$

According to two regions A and B in Figure 3, the ratio of thermal cloaking (RTC) can be calculated as follows:

$$
R T C=\frac{J_{A}}{J_{B}}
$$

where $J$ is the average heat flux. In addition, we can also calculate the single-atom temperature during the simulation as follows:

$$
\sum_{i} \varepsilon_{i}=\frac{d i m}{2} k_{B} N T
$$

where $\varepsilon$ is the kinetic energy, $i$ is an atomic label, $\operatorname{dim}$ is the dimensionality $(\operatorname{dim}=3), k_{B}$ is the Boltzmann constant, and $N$ is the total number of atoms.

\subsubsection{Phonon Localization Theory}

Phonon localization theory has important applications in various fields. In a related study on graphene [57-59], with the help of phonon localization theory, it was found that phonon localization mainly affects long-wave and high-frequency modes, and it was found that the strong localization of low-frequency phonons caused by reverse short thermal contacts leads to a clear temperature transition. In the study of heterostructures [60,61], with the help of phonon localization theory, the internal mechanism of the abnormal interfacial thermal conductance (ITC) change at the interface of Gr and h-BN was explored, aiming at the topological defect-based semi-defective graphene/hexagonal boron nitride. With respect to the thermal conductivity of the in-plane heterostructure interface, it was found that the phonon coupling on both sides of the interface and the phonon localization effect of the heterostructure were the two keys determining the heterostructure ITC factor. In other fields [62], the effect of anti-substitution on thermal conductivity was investigated using nonequilibrium molecular dynamics simulations, and it was found that, when the defect concentration was low, localization was the main reason for the decrease in thermal conductivity, whereas, when the defect concentration was high, the main reason was phonon defect scattering in all phonon modes.

With the help of the phonon localization theory, we can explain the cloaking mechanism by calculating the phonon density of states (PDOS). To make the results meaningful, we selected the same region in the cloak and the crystalline film. Since the cloaking phenomenon is caused by the existence of the functional region, we chose the whole functional region as the calculation region. PDOS can be described as

$$
\operatorname{PDOS}(\omega)=\frac{1}{N \sqrt{2 \pi}} \int e^{-i \omega t}\left\langle\sum_{j=1}^{N} \mathbf{v}_{j}(0) \mathbf{v}_{j}(t)\right\rangle d t,
$$

where $N$ is the total number of atoms, $\omega$ is the phonon frequency, $\mathbf{v}$ is the velocity vector, and $j$ is an atomic label.

To better understand the PDOS, the mode participation rate (MPR) can be calculated as follows [63]:

$$
\operatorname{MPR}(\omega)=\frac{1}{N} \frac{\left[\sum_{i} \operatorname{PDOS}_{i}(\omega)^{2}\right]^{2}}{\sum_{i} \operatorname{PDOS}_{i}(\omega)^{4}},
$$

where $N$ is the total number of atoms, and $\operatorname{PDOS}_{i}(\omega)$ is local density of states based on Equation (9). 


\subsection{Simulation Process}

We used NEMD to calculate the thermal conductivity of the constructed model. This method requires a heat source and a heat sink to be applied to the model, both of which were $0.0005 \mathrm{eV} \cdot \AA / \mathrm{ps}$ in this paper. The initial temperature of the whole system was fixed at $300 \mathrm{~K}$, and the simulation was run for $10 \mathrm{~ns}$.

We then simulated the thermal cloak. The timestep was $1 \mathrm{fs}$. Periodic boundary conditions were used in all three directions during the simulation. The whole process was divided into two stages. In the preparation stage, the energy of the whole system was first minimized, and all atoms except the fixed region were given an initial velocity satisfying a Gaussian distribution at a temperature of $300 \mathrm{~K}$ (achieved by specifying a random number in LAMMPS; the random number in this paper was 4,928,459). In the simulation stage, the selected calculated system was first placed in the NVT ensemble, simulated for 100 ps at $300 \mathrm{~K}$ for full relaxation, and then placed in the NVE ensemble and simulated for $1000 \mathrm{ps}$. The hot bath and cold bath were $350 \mathrm{~K}$ and $250 \mathrm{~K}$, respectively, and the thermostat used was Nose-Hoover [64].

$$
\begin{gathered}
\frac{d}{d t} p_{i}=F_{i}-\gamma p_{i}, \\
\frac{d}{d t} \gamma=\frac{1}{\tau^{2}}\left[\frac{T(t)}{T_{0}}-1\right], \\
T(t)=\frac{2}{3 N k_{B}} \sum_{i} \frac{p_{i}^{2}}{2 m_{i}},
\end{gathered}
$$

where $i$ is an atomic label, $p$ is the momentum, $F$ is the force, $\gamma$ is a dynamic parameter, $\tau$ is the relaxation time, $m$ is the mass, $k_{B}$ is the Boltzmann constant, and $N$ is the number of atoms in the thermostats.

\section{Results and Discussion}

\subsection{The Thermal Conductivity}

We obtained the temperature change curve through simulation and fit it to obtain the slope of the curve, as shown in Figure 4. The slope was $\nabla T$. Using Equation (2), we calculated the thermal conductivity. The results prove that the thermal conductivity of the porous structure was lower than that of bulk silicon; hence, it could be used to construct the nanocloak.

(a)

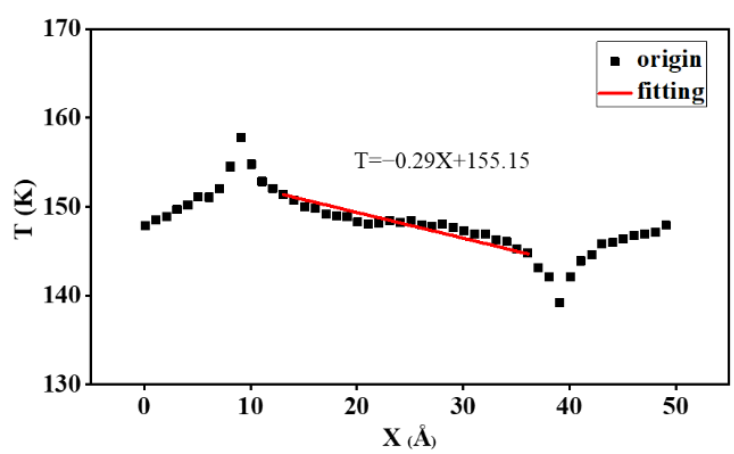

(b)

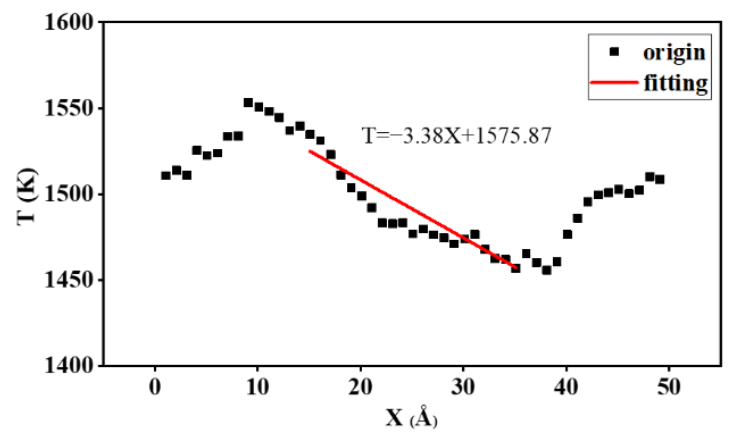

Figure 4. Temperature of the calculated model: (a) crystalline silicon; (b) porous silicon structure.

\subsection{The Calculation of Thermal Cloak Performance}

Figure 5 shows the RTC of the thermal cloak and crystalline silicon film at 600 ps. The RTC of the thermal cloak was much higher than that of the crystalline silicon film, confirming the cloaking phenomenon, and the cloaking effect was obvious. Compared with bulk silicon, the porous silicon structure had a large porosity, which was not conducive to the transmission of phonons, resulting in low thermal conductivity and producing the 
cloaking phenomenon. Increasing the porosity of the porous structure could produce excellent cloaking effects.

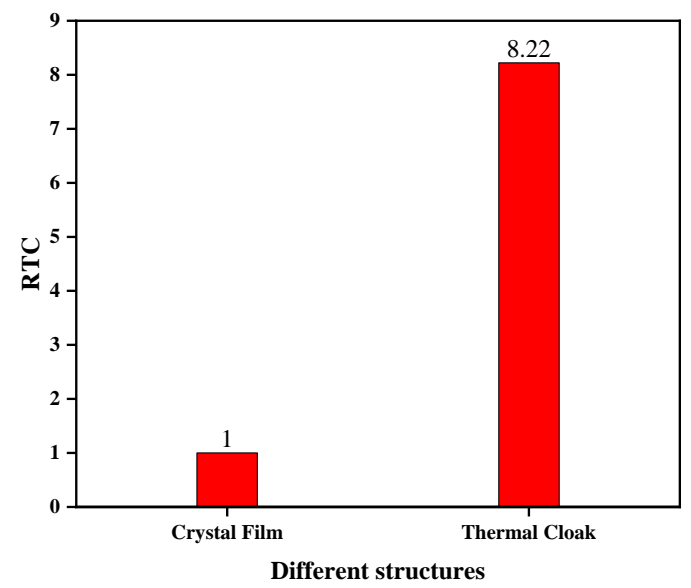

Figure 5. The RTC with different structures at $600 \mathrm{ps}$.

To prove the occurrence of the cloaking phenomenon, the heat flux and temperature distribution of the crystalline film and the nanocloak at $600 \mathrm{ps}$ are plotted in Figure 6. In the simulation process, we calculated the heat flux and temperature of a single atom; however, we do not care about the state of a single atom but the state of the whole system. Therefore, we first divided the whole system into 40 small blocks, and then calculated the average heat flux and average temperature in each small block. By analyzing the heat flux distribution, we can clearly see the cloaking phenomenon. The heat flux of the whole functional region of the thermal cloak was kept extremely low, and the heat flux was spread around the functional region. However, when observing the temperature distribution, the cloaking phenomenon was not obvious, because the nanocloak is a typical heat flux control device, rather than a temperature control device.

\subsection{Analysis of Cloaking Mechanism of Nanocloak}

Figure 7 shows the PDOS of the crystalline silicon film and the thermal cloak. For the crystalline silicon film, consistent with a previous study [65], a weak and a strong peak appeared at $5 \mathrm{THz}$ and $17 \mathrm{THz}$, respectively. Phonons are generated due to the excitation of atomic vibration. For the same atom, the vibration frequency was the same, and the phonons excited by the vibration were roughly the same. For the thermal cloak, due to the reduction in the number of atoms in the functional region, both the low- and the highfrequency peaks shifted to the left, and the PDOS was larger than the crystalline silicon film in the range of $0-5 \mathrm{THz}$, while the PDOS was smaller than the crystalline silicon film in the range of 15.5-18.5 THz. In addition, a large number of phonon modes appeared in the range of $5-15 \mathrm{THz}$.

Figure 8 shows the MPR in the calculated region of the different structures. For the crystalline silicon film, the MPR was uniformly distributed, basically greater than 0.6 , indicating that these phonon modes were decentralized. For the thermal cloak, all MPRs were lower than 0.6, and the distribution was uneven, proving the occurrence of the phonon localization phenomenon [66]. The main reason is that the number of atoms in the functional region of the thermal cloak was reduced, while there were fewer phonons excited by vibration in the same region, and the porous structure was not conducive to the propagation of phonons, which in turn affected the PDOS. The PDOS at low frequency increased, and the peak shifted to the left, while the PDOS at high frequency decreased, and the peak shifted to the left, resulting in a strong phonon localization, which in turn reduced the thermal conductivity of the functional region, resulting in the cloaking phenomenon. 
(a)
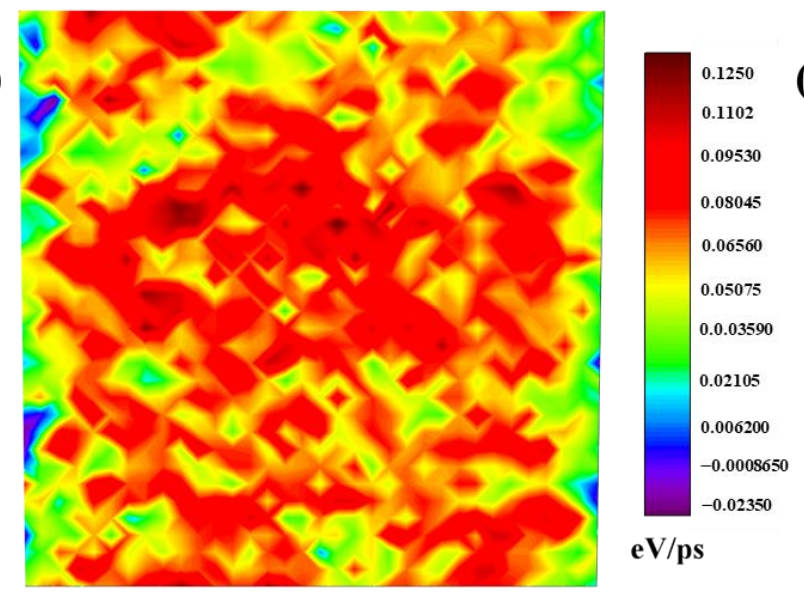

(c)

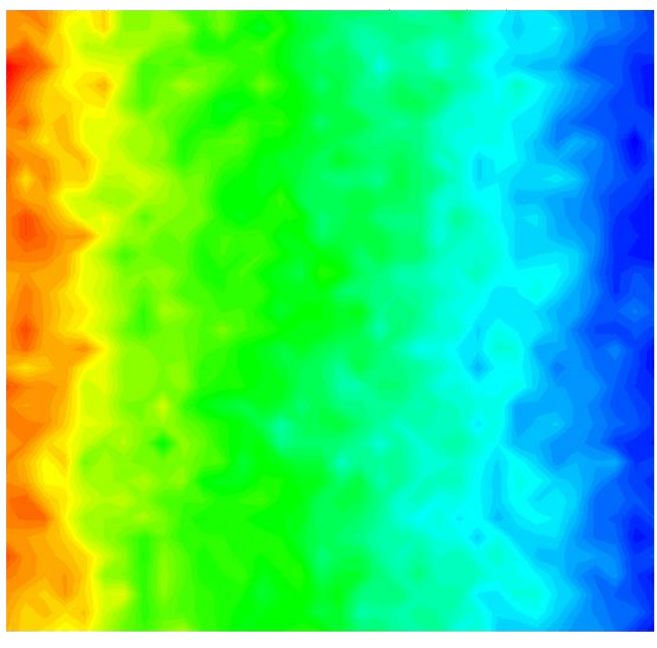

(b)
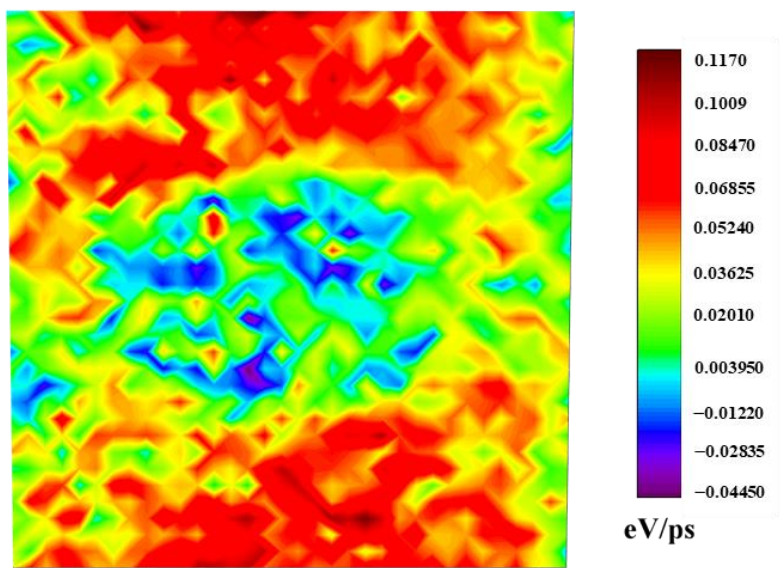

eV/ps

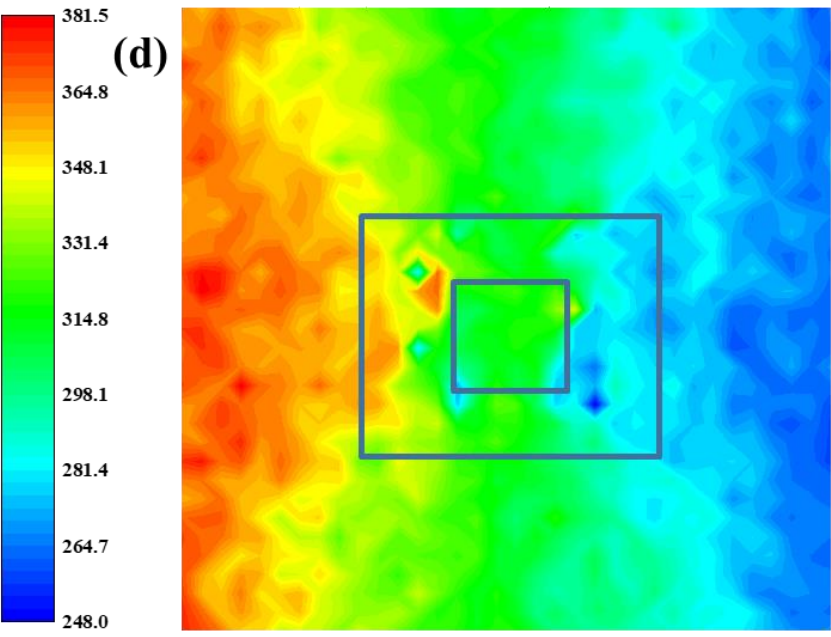

T/K

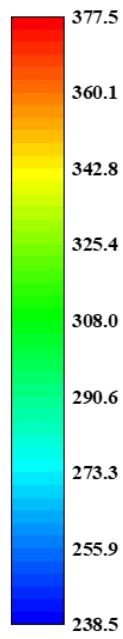

T/K

Figure 6. (a,b) The heat flux distribution at $600 \mathrm{ps,} \mathrm{where} \mathrm{(a)} \mathrm{is} \mathrm{the} \mathrm{crystalline} \mathrm{film,} \mathrm{and} \mathrm{(b)} \mathrm{is} \mathrm{the}$ nanocloak; (c,d) the temperature distribution at 600 ps, where (c) is the crystalline film, and (d) is the nanocloak.

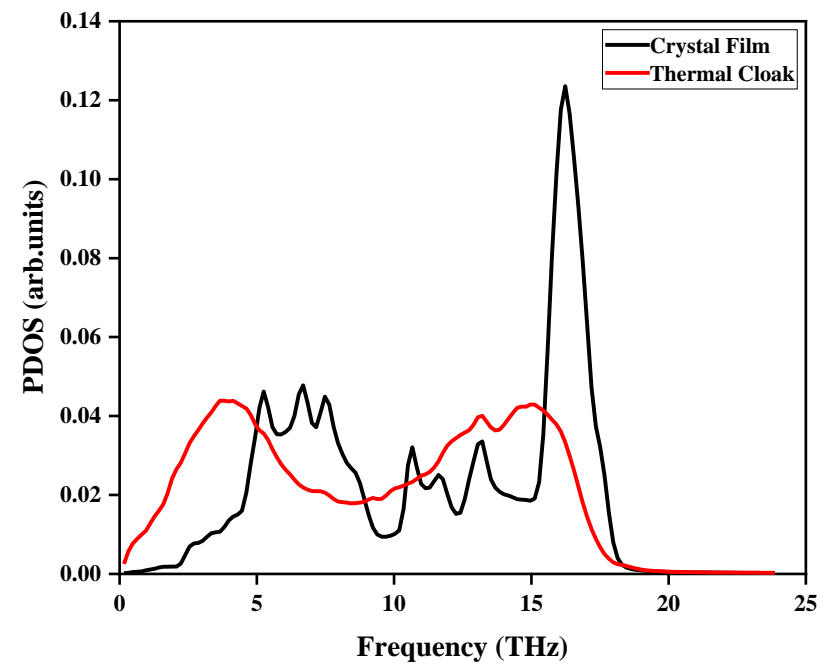

Figure 7. PDOS in the calculated region of the different structures. 


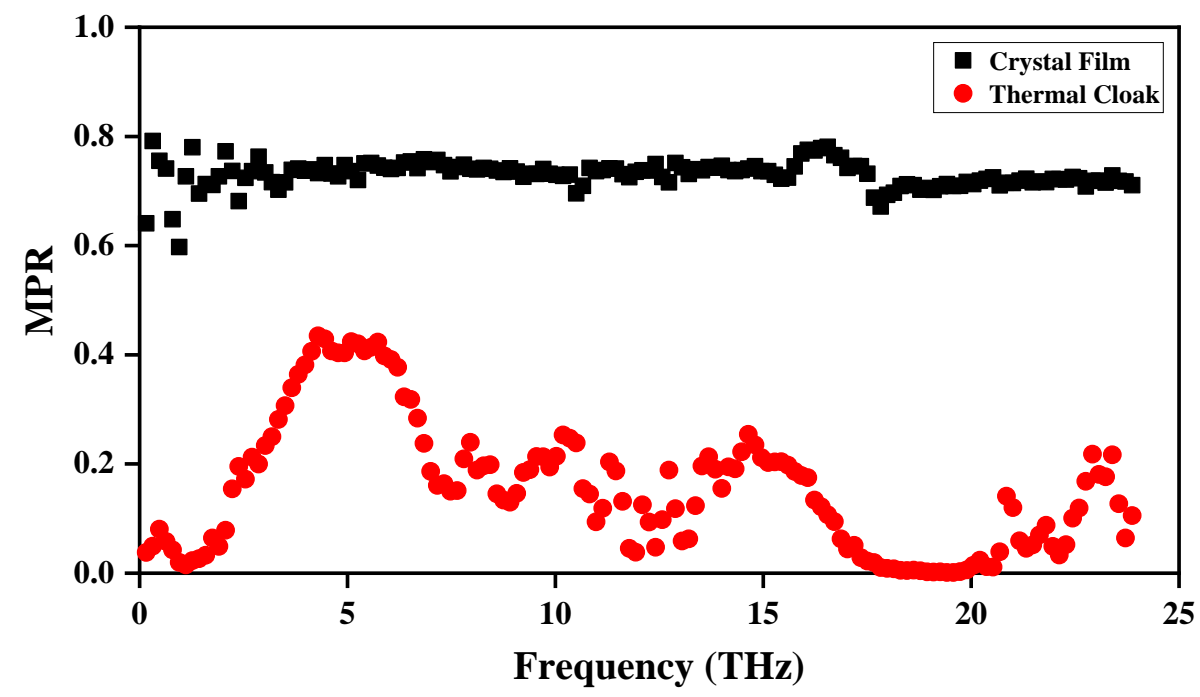

Figure 8. MPR in the calculated region of the different structures.

\section{Conclusions}

In this paper, we used LAMMPS software for modeling and simulation, and we constructed thermal conductivity and thermal cloak calculation models. The RTC was used to evaluate the cloaking performance, and the phonon localization theory was used to explain its cloaking mechanism. This research has important applications in the thermal isolation of microelectronic devices, expands the construction methods of thermal cloaks, and promotes the application of porous materials. The main conclusions are summarized as follows:

(1) Through NEMD simulation, we found that the thermal conductivity of the porous structure was significantly lower than that of the crystalline film, satisfying the conditions for building the thermal cloak;

(2) The RTC was used to evaluate the cloaking performance, proving that the nanocloak built with a porous structure could produce excellent cloaking effects, which was verified by drawing the heat flux distribution;

(3) The cloaking mechanism of the nanocloak was analyzed by phonon localization theory; we calculated the PDOS and MPR, and we found that due to the reduction in the number of atoms in the functional region of the thermal cloak, the phonons generated by vibration were reduced, while the porous structure was not conducive to the propagation of phonons, which in turn affected the PDOS. The PDOS at low frequency increased, and the peak shifted to the left, while the PDOS at high frequency decreased, and the peak shifted to the left, resulting in a strong phonon localization. As a result, the thermal conductivity of the functional region of the thermal cloak was reduced, and the phenomenon of cloaking occurred.

In this paper, it was pointed out that an increase in porosity of the porous structure led to a decrease in thermal conductivity, but the effect of porosity on the cloaking performance of the nanocloak was not explored, as this paper only explored the cloaking performance of the porous cloak within a constant temperature boundary. In order to facilitate engineering applications, the dynamic environment response is also critical, and we will focus on addressing these issues in the future to advance the application of porous structures in the field of heat flow regulation.

Author Contributions: Conceptualization, J.Z. and H.Z.; methodology, J.Z.; software, J.Z.; validation, J.Z., Y.L. and Q.W.; data curation, W.S. and Y.L.; writing-original draft preparation, J.Z.; visualization, Q.W. and W.S.; supervision, H.Z. All authors have read and agreed to the published version of the manuscript. 
Funding: This research was funded by the National Natural Science Foundation of China, grant number 51776050 .

Institutional Review Board Statement: Not applicable.

Informed Consent Statement: Not applicable.

Data Availability Statement: The data presented in this study are available in this article.

Conflicts of Interest: The authors declare no conflict of interest.

\section{References}

1. Fan, C.Z.; Gao, Y.; Huang, J.P. Shaped graded materials with an apparent negative thermal conductivity. Appl. Phys. Lett. 2008, 92, 251907. [CrossRef]

2. Han, T.C.; Zhao, J.J.; Yuan, T.; Lei, D.Y.; Li, B.W.; Qiu, C.W. Theoretical realization of an ultra-efficient thermal-energy harvesting cell made of natural materials. Energy Environ. Sci. 2013, 6, 3537-3541. [CrossRef]

3. Yang, T.Z.; Huang, L.J.; Chen, F.; Xu, W.K. Heat flux and temperature field cloaks for arbitrarily shaped objects. J. Phys. D Appl. Phys. 2013, 46, 305102. [CrossRef]

4. Han, T.C.; Bai, X.; Gao, D.L.; Thong, J.T.L.; Li, B.W.; Qiu, C.W. Experimental Demonstration of a Bilayer Thermal Cloak. Phys. Rev. Lett. 2014, 112, 054302. [CrossRef] [PubMed]

5. Nguyen, D.M.; Xu, H.G.; Zhang, Y.M.; Zhang, B.L. Active thermal cloak. Appl. Phys. Lett. 2015, 107, 121901. [CrossRef]

6. Han, T.C.; Bai, X.; Liu, D.; Gao, D.L.; Li, B.W.; Thong, J.T.L.; Qiu, C.W. Manipulating Steady Heat Conduction by Sensu-shaped Thermal Metamaterials. Sci. Rep. 2015, 5, 10242. [CrossRef] [PubMed]

7. Yang, T.Z.; Bai, X.; Gao, D.L.; Wu, L.Z.; Li, B.W.; Thong, J.T.L.; Qiu, C.W. Invisible Sensors: Simultaneous Sensing and Camouflaging in Multiphysical Fields. Adv. Mater. 2015, 27, 7752-7758. [CrossRef]

8. $\quad$ Li, T.H.; Zhu, D.L.; Mao, F.C.; Huang, M.; Yang, J.J.; Li, S.B. Design of diamond-shaped transient thermal cloaks with homogeneous isotropic materials. Front. Phys. 2016, 11, 110503. [CrossRef]

9. Shen, X.Y.; Li, Y.; Jiang, C.R.; Ni, Y.S.; Huang, J.P. Thermal cloak-concentrator. Appl. Phys. Lett. 2016, 109, 031907. [CrossRef]

10. Zhou, L.L.; Huang, S.Y.; Wang, M.; Hu, R.; Luo, X.B. While rotating while cloaking. Phys. Lett. A 2019, 383, 759-763. [CrossRef]

11. Zhu, Z.; Ren, X.C.; Sha, W.; Xiao, M.; Hu, R.; Luo, X.B. Inverse design of rotating metadevice for adaptive thermal cloaking. Int. J. Heat Mass Transf. 2021, 176, 121417. [CrossRef]

12. Hu, R.; Wei, X.L.; Hu, J.Y.; Luo, X.B. Local heating realization by reverse thermal cloak. Sci. Rep. 2014, 4, 3600. [CrossRef]

13. Chen, F.; Lei, D.Y. Experimental Realization of Extreme Heat Flux Concentration with Easy-to-Make Thermal Metamaterials. Sci. Rep. 2015, 5, 11552. [CrossRef]

14. Guenneau, S.; Amra, C. Anisotropic conductivity rotates heat fluxes in transient regimes. Opt. Express 2013, 21, 6578-6583. [CrossRef]

15. Han, T.C.; Bai, X.; Thong, J.T.L.; Li, B.W.; Qiu, C.W. Full Control and Manipulation of Heat Signatures: Cloaking, Camouflage and Thermal Metamaterials. Adv. Mater. 2014, 26, 1731-1734. [CrossRef] [PubMed]

16. Yang, T.Z.; Su, Y.S.; Xu, W.K.; Yang, X.D. Transient thermal camouflage and heat signature control. Appl. Phys. Lett. 2016, 109, 121905. [CrossRef]

17. Wang, J.; Bi, Y.Q.; Hou, Q.W. Three-dimensional illusion thermal device for location camouflage. Sci. Rep. 2017, 7, 7541. [CrossRef]

18. Li, Y.; Bai, X.; Yang, T.Z.; Luo, H.L.; Qiu, C.W. Structured thermal surface for radiative camouflage. Nat. Commun. 2018, 9, 273. [CrossRef]

19. Qu, Y.R.; Li, Q.; Cai, L.; Pan, M.Y.; Ghosh, P.; Du, K.K.; Qiu, M. Thermal camouflage based on the phase-changing material GST. Light Sci. Appl. 2018, 7, 26. [CrossRef] [PubMed]

20. Hu, R.; Xi, W.; Liu, Y.D.; Tang, K.C.; Song, J.L.; Luo, X.B.; Wu, J.Q.; Qiu, C.W. Thermal camouflaging metamaterials. Mater. Today 2020, 45, 120-141. [CrossRef]

21. Liu, Y.D.; Song, J.L.; Zhao, W.X.; Ren, X.C.; Cheng, Q.; Luo, X.B.; Fang, N.X.; Hu, R. Dynamic thermal camouflage via a liquid-crystal-based radiative metasurface. Nanophotonics 2020, 9, 855-863. [CrossRef]

22. Zhu, N.Q.; Shen, X.Y.; Huang, J.P. Converting the patterns of local heat flux via thermal illusion device. AIP Adv. 2015, 5, 053401. [CrossRef]

23. Alwakil, A.; Zerrad, M.; Bellieud, M.; Amra, C. Inverse heat mimicking of given objects. Sci. Rep. 2017, 7, 43288. [CrossRef]

24. Hu, R.; Zhou, S.L.; Li, Y.; Lei, D.Y.; Luo, X.B.; Qiu, C.W. Illusion Thermotics. Adv. Mater. 2018, 30, 1707237. [CrossRef]

25. Zhou, S.L.; Hu, R.; Luo, X.B. Thermal illusion with twinborn-like heat signatures. Int. J. Heat Mass Transf. 2018, 127, 607-613. [CrossRef]

26. Hu, R.; Huang, S.Y.; Wang, M.; Luo, X.B.; Shiomi, J.; Qiu, C.W. Encrypted Thermal Printing with Regionalization Transformation. Adv. Mater. 2019, 31, 1807849. [CrossRef] [PubMed]

27. Xu, G.Q.; Zhang, H.C.; Zhang, X.; Jin, Y. Investigating the thermodynamic performances of TO-based metamaterial tunable cells with an entropy generation approach. Entropy 2017, 19, 538. [CrossRef]

28. Xu, G.Q.; Zhang, H.C.; Zou, Q.; Jin, Y. Predicting and analyzing interaction of the thermal cloaking performance through response surface method. Int. J. Heat Mass Transf. 2017, 109, 746-754. [CrossRef] 
29. Li, Y.Y.; Zhang, H.C.; Sun, M.Y.; Zhang, Z.H.; Zhang, H.M. Environmental Response of 2D Thermal Cloak under Dynamic External Temperature Field. Entropy 2020, 22, 461. [CrossRef]

30. Xu, G.Q.; Zhou, X.; Zhang, H.C.; Tan, H.P. Creating illusion of discrete source array by simultaneously allocating thermal and DC fields with homogeneous media. Energy Convers. Manag. 2019, 187, 546-553. [CrossRef]

31. Li, Y.Y.; Zhang, H.C.; Chen, Y.J.; Zhang, D.; Huang, Z.L.; Wang, H.M. Realization and analysis of an Intelligent flux transfer regulator by allocating thermal and DC electric fields. Int. J. Heat Mass Transf. 2021, 179, 121677. [CrossRef]

32. Liu, Y.Z.; Xu, Y.; Zhang, S.C.; Duan, W.H. Model for topological phononics and phonon diode. Phys. Rev. B 2017, 96, 064106. [CrossRef]

33. Wang, S.; Cottrill, A.L.; Kunai, Y.; Toland, A.R.; Liu, P.W.; Wang, W.J.; Strano, M.S. Microscale solid-state thermal diodes enabling ambient temperature thermal circuits for energy applications. Phys. Chem. Chem. Phys. 2017, 19, 13172-13181. [CrossRef]

34. Zhang, L.F.; Ren, J.; Wang, J.S.; Li, B.W. Topological Nature of the Phonon Hall Effect. Phys. Rev. Lett. 2010, 105, 225901. [CrossRef]

35. Qin, T.; Zhou, J.H.; Shi, J.R. Berry curvature and the phonon Hall effect. Phys. Rev. B 2012, 86, 104305. [CrossRef]

36. Li, N.B.; Ren, J.; Wang, L.; Zhang, G.; Haenggi, P.; Li, B.W. Colloquium: Phononics: Manipulating heat flow with electronic analogs and beyond. Rev. Mod. Phys. 2012, 84, 1045-1066. [CrossRef]

37. Chang, C.W.; Okawa, D.; Majumdar, A.; Zettl, A. Solid-state thermal rectifier. Science 2006, 314, 1121-1124. [CrossRef] [PubMed]

38. Hu, J.N.; Ruan, X.L.; Chen, Y.P. Molecular Dynamics Study of Thermal Rectification in Graphene Nanoribbons. Int. J. Thermophys. 2012, 33, 986-991. [CrossRef]

39. Tian, H.; Xie, D.; Yang, Y.; Ren, T.L.; Zhang, G.; Wang, Y.F.; Zhou, C.J.; Peng, P.G.; Wang, L.G.; Liu, L.T. A Novel Solid-State Thermal Rectifier Based on Reduced Graphene Oxide. Sci. Rep. 2012, 2, 523. [CrossRef]

40. Wang, Y.; Vallabhaneni, A.; Hu, J.N.; Qiu, B.; Chen, Y.P.; Ruan, X.L. Phonon Lateral Confinement Enables Thermal Rectification in Asymmetric Single-Material Nanostructures. Nano Lett. 2014, 14, 592-596. [CrossRef] [PubMed]

41. Ye, Z.Q.; Cao, B.Y. Nanoscale thermal cloaking in graphene via chemical functionalization. Phys. Chem. Chem. Phys. 2016, 18, 32952-32961. [CrossRef]

42. Liu, Y.D.; Cheng, Y.H.; Hu, R.; Luo, X.B. Nanoscale thermal cloaking by in-situ annealing silicon membrane. Phys. Lett. A 2019, 383, 2296-2301. [CrossRef]

43. Choe, H.S.; Prabhakar, R.; Wehmeyer, G.; Allen, F.; Lee, W.; Jin, L.; Li, Y.; Yang, P.D.; Qiu, C.W.; Dames, C.; et al. Ion Write Microthermotics: Programing Thermal Metamaterials at the Microscale. Nano Lett. 2019, 19, 3830-3837. [CrossRef]

44. Zhang, J.; Zhang, H.C.; Li, Y.Y.; Zhang, D.; Wang, H.M. Numerical analysis on nanoscale thermal cloak in three-dimensional silicon film with circular cavities. Numer. Heat Transf. Part A Appl. 2022, 81, 1-14. [CrossRef]

45. Zhang, J.; Zhang, H.C.; Sun, W.B.; Wang, Q. Mechanism analysis of double-layer nanoscale thermal cloak by silicon film. Colloids Surf. A Physicochem. Eng. Asp. 2022, 634, 128022. [CrossRef]

46. Zhang, J.; Zhang, H.; Wang, H.; Xu, C.; Wang, Q. Performance prediction of nanoscale thermal cloak by molecular dynamics. Appl. Phys. A 2021, 127, 790. [CrossRef]

47. Xiao, Y.; Chen, Q.Y.; Hao, Q. Inverse thermal design of nanoporous thin films for thermal cloaking. Mater. Today Phys. 2021, 21, 100477. [CrossRef]

48. Chen, G.; Dresselhaus, M.S.; Dresselhaus, G.; Fleutial, J.P.; Caillat, T. Recent developments in thermoelectric materials. Int. Mater Rev. 2003, 48, 45-66. [CrossRef]

49. Plimpton, S. Fast Parallel Algorithms for Short-Range Molecular Dynamics. J. Comput. Phys. 1995, 117, 1-19. [CrossRef]

50. Zhang, Y.H.; Feller, S.E.; Brooks, B.R.; Pastor, R.W. Computer simulation of liquid/liquid interfaces. I. Theory and application to octane/water. J. Chem. Phys. 1995, 103, 10252-10266. [CrossRef]

51. Tersoff, J. Modeling solid-state chemistry: Interatomic potentials for multicomponent systems. Phys. Rev. B 1989, 39, 5566-5568. [CrossRef] [PubMed]

52. Liu, B.K.; Vu-Bac, N.; Rabczuk, T. A stochastic multiscale method for the prediction of the thermal conductivity of Polymer nanocomposites through hybrid machine learning algorithms. Compos. Struct. 2021, 273, 114269. [CrossRef]

53. Rabizadeh, E.; Bagherzadeh, A.S.; Anitescu, C.; Alajlan, N.; Rabczuk, T. Pointwise dual weighted residual based goal-oriented a posteriori error estimation and adaptive mesh refinement in 2D/3D thermo-mechanical multifield problems. Comput. Methods Appl. Mech. Eng. 2020, 359, 112666. [CrossRef]

54. Vu-Bac, N.; Duong, T.X.; Lahmer, T.; Zhang, X.; Sauer, R.A.; Park, H.S.; Rabczuk, T. A NURBS-based inverse analysis for reconstruction of nonlinear deformations of thin shell structures. Comput. Methods Appl. Mech. Eng. 2018, 331, 427-455. [CrossRef]

55. Rabczuk, T.; Ren, H.L.; Zhuang, X.Y. A Nonlocal Operator Method for Partial Differential Equations with Application to Electromagnetic Waveguide Problem. Comput. Mater. Contin. 2019, 59, 31-55. [CrossRef]

56. Chen, X.K.; Hu, J.W.; Wu, X.J.; Jia, P.; Peng, Z.H.; Chen, K.Q. Tunable Thermal Rectification in Graphene/Hexagonal Boron Nitride Hybrid Structures. J. Phys. D 2018, 51, 396-400. [CrossRef]

57. Loh, G.C.; Teo, E.H.T.; Tay, B.K. Phonon localization around vacancies in graphene nanoribbons. Diam. Relat. Mater. 2012, 23, 88-92. [CrossRef]

58. Jiang, P.F.; Hu, S.Q.; Ouyang, Y.L.; Ren, W.J.; Ren, C.Q.; Zhang, Z.W.; Chen, J. Remarkable thermal rectification in pristine and symmetric monolayer graphene enabled by asymmetric thermal contact. J. Appl. Phys. 2020, 127, 235101. [CrossRef]

59. Lu, S.; Ouyang, Y.L.; Yu, C.Q.; Jiang, P.F.; He, J.; Chen, J. Tunable phononic thermal transport in two-dimensional C6CaC6 via guest atom intercalation. J. Appl. Phys. 2021, 129, 225106. [CrossRef] 
60. Liang, T.; Zhou, M.; Zhang, P.; Yuan, P.; Yang, D.G. Multilayer in-plane graphene/hexagonal boron nitride heterostructures: Insights into the interfacial thermal transport properties. Int. J. Heat Mass Transf. 2020, 151, 119395. [CrossRef]

61. Wu, X.; Han, Q. Thermal transport in pristine and defective two-dimensional polyaniline (C3N). Int. J. Heat Mass Transf. 2021, 173, 121235. [CrossRef]

62. Zhou, M.; Liang, T.; Wu, B.Y.; Liu, J.J.; Zhang, P. Phonon transport in antisite-substituted hexagonal boron nitride nanosheets: A molecular dynamics study. J. Appl. Phys. 2020, 128, 234304. [CrossRef]

63. Loh, G.C.; Teo, E.H.T.; Tay, B.K. Phononic and structural response to strain in wurtzite-gallium nitride nanowires. J. Appl. Phys. 2012, 111, 103506. [CrossRef]

64. Berendsen, H.J.C.; Postma, J.P.M.; van Gunsteren, W.F.; DiNola, A.; Haak, J.R. Molecular dynamics with coupling to an external bath. J. Chem. Phys. 1984, 81, 3684-3690. [CrossRef]

65. Li, H.P.; Zhang, R.Q. Anomalous effect of hydrogenation on phonon thermal conductivity in thin silicon nanowires. Eur. Lett. 2014, 105, 56003. [CrossRef]

66. Ma, D.K.; Ding, H.R.; Meng, H.; Feng, L.; Wu, Y.; Shiomi, J.; Yang, N. Nano-cross-junction effect on phonon transport in silicon nanowire cages. Phys. Rev. B 2016, 94, 165434. [CrossRef] 